Pacific Journal of Mathematics

LARGE INDECOMPOSABLE CONTINUA WITH ONLY ONE
COMPOSANT 


\title{
LARGE INDECOMPOSABLE CONTINUA WITH ONLY ONE COMPOSANT
}

\author{
Michel SMith
}

\begin{abstract}
David P. Bellamy has shown that there exist indecomposable Hausdorff continua with only one or only two composants. The continua that he constructs are small in the sense that they do not have more than $2^{c}$ points. In this paper his results are generalized; in particular it will be shown that if $X$ is a Hausdorff continuum then $X$ is a retract of an indecomposable continuum with exactly one composant and of an indecomposable continuum with exactly two composants.
\end{abstract}

Definitions and Notations. A continuum is a compact connected เusdorff space. Suppose $\lambda$ is an ordinal, $I_{a}$ is a topological space for each $a<\lambda$, and if $a<b$ then $r_{a}^{b}$ is a mapping from $I_{b}$ onto $I_{a}$ so that if $a<b<c<\lambda$ then $r_{a}^{b} \circ r_{b}^{c}=r_{a}^{c}$. Then the space $I=$ $\lim \left\{I_{a}, r\right\}_{a<\lambda}$ denotes the space which is the inverse limit of the inverse system $\left\{I_{a}, r_{a}^{b}\right\}_{a<b<\lambda}$. Each point $P$ of $I$ is a function from $\lambda$ into $\bigcup_{a<\lambda} I_{a}$ such that $P_{a} \in I_{a} . \quad \Pi_{a}$ denotes the function from $I$ into $I_{a}$ such that $\Pi_{a}(P)=P_{a}$. If $R \subset I_{a}$ then $\overleftarrow{R}=\left\{x \mid x_{a} \in R\right\}$. If $S=\Pi_{a \in A} S_{a}$ is a product space then $x=\left\{x_{a}\right\}_{a \in A}$ denotes a point of $S$ so that $x_{a} \in S_{a}$ and $\pi_{a}$ denotes the function from $S$ into $S_{a}$ so that $\pi_{a}(x)=x_{a}$. The composant of the continuum $M$ containing the point $P$ of $M$ is the set of points $Q$ of $M$ such that there is a proper subcontinuum of $M$ containing $P$ and $Q$, it is denoted by $\operatorname{Cmps}(M, P)$.

Construction. The following construction employs techniques used in [1] and [4]. The continuum will be constructed as an inverse limit $\lim \left\{I_{a}, r\right\}_{a<\omega_{1}}$ such that for each $a<\omega_{1} I_{a}$ is a subset of the cartesian product of $I_{0}$ and $\omega_{1}$ copies of $[0,1]$ so that if $b$ is an ordinal with $a<b<\omega_{1}$ then $I_{a}$ will be homeomorphic to a subset of $I_{b}$; in fact it will be convenient to identify $I_{a}$ with this subset so that $\left\{I_{a}\right\}_{a<\omega_{1}}$ will be a monotonic collection of continua, $I_{a}$ may be considered to be a subset of $I_{0} \times \Pi_{i \leqq a}[0,1] \times \Pi_{a<j<\omega_{1}}\{0\}$, and if $x \in I_{a+1}$ then $\pi_{a+1}(x) \in[0,1], \pi_{j}(x)=0$ if $j>a+1$, and $\Pi_{i<a+1}\left\{\pi_{i}(x)\right\} \times$ $\Pi_{j>a+1}\{0\}$ is a point of $I_{a}$. In general the space $\Pi_{j<a}[0,1]$ may be considered to be the space $\Pi_{j<a}[0,1] \times \Pi_{a<i<\omega_{1}}\{0\}$.

Construction of $I_{0}$ : If $X$ is a continuum then there exists a continuum $I_{0}$ containing $X$ as a retract which is irreducible from some point $1_{0}$ to $X$ so that: there exists a sequence of points $\left\{a_{1}^{0}\right\}_{i=1}^{\infty}$ and a monotonic sequence of proper subcontinua of $I_{0},\left\{A_{i}^{0}\right\}_{i=1}^{\infty}$ such that 
(1) $\left\{a_{i}^{0}\right\}_{i=1}^{\infty}$ converges to a point $a$ in $X$, (2) $A_{i}^{0}$ is irreducible from $1_{0}$ to $a_{i}^{0}$ and $A_{i}^{0} \subset A_{i+1}^{0}$ for each positive integer $i$, and (3) Cmps $\left(I_{0}, 1_{0}\right)=$ $\bigcup_{i=1}^{\infty} A_{i}^{0}$. (The existence of $I_{0}$ follows from [4] or from [2] and the construction for $I_{1}$ used below.)

Construction of $I_{1}$ : Let $I_{1}$ be the subcontinuum of $I_{0} \times[0,1]$ defined as follows: for each positive integer $n$ let $a_{n}^{1}=\left(a_{n}^{0}, 1 /(2 n-1)\right)$,

$$
\begin{aligned}
& A_{1}^{1}=A_{1}^{0} \times\{1\}, \\
& A_{2}^{1}=A_{1}^{1} \cup\left(\left\{a_{1}^{0}\right\} \times[1 / 2,1]\right) \cup\left(A_{1}^{0} \times\{1 / 2\}\right) \\
& \cup\left(\left\{1_{0}\right\} \times\left[\frac{1}{3}, 1 / 2\right]\right) \cup\left(A_{2}^{0} \times\left\{\frac{1}{3}\right\}\right), \\
& A_{n}^{1}=A_{n-1}^{1} \cup\left(\left\{a_{n-1}^{0}\right\} \times\left[\frac{1}{2 n-2}, \frac{1}{2 n-3}\right]\right) \\
& \cup\left(\left\{A_{n-1}^{0}\right\} \times\left\{\frac{1}{2 n-2}\right\}\right) \cup\left(\left\{1_{0}\right\} \times\left[\frac{1}{2 n-1}, \frac{1}{2 n-2}\right]\right) \\
& \cup\left(A_{n}^{0} \times\left\{\frac{1}{2 n-1}\right\}\right),
\end{aligned}
$$

and let $I_{1}=\left(I_{0} \times\{0\}\right) \cup \bigcup_{n=1}^{\infty} A_{n}^{1}$. Let $1_{1}=\left(1_{0}, 1\right)$ and identify $I_{0}$ with $I_{0} \times\{0\}$ using the natural mapping. Thus $\left\{a_{n}^{1}\right\}_{n=1}^{\infty}$ converges to $(a, 0)$ which has been identified with $a, A_{n}^{1}$ is irreducible from $a_{n}^{1}$ to $1_{1}$, and $A_{n}^{1} \subset A_{n+1}^{1}$. Let $r_{0}^{1}$ be the projection $\pi_{1}$ of $I_{1}$ onto $I_{0}$, thus $r_{0}^{1}\left(A_{n}^{1}\right)=$ $A_{n}^{0}, r_{0}^{1}\left(a_{n}^{1}\right)=a_{n}^{0}$, and $\operatorname{Cmps}\left(I_{1}, 1_{1}\right)=\bigcup_{n=1}^{\infty} A_{n}^{1}$.

Construction of $I_{k}$ for each positive integer $k>1$ : Let $I_{k}$ be a subcontinuum of $I_{k-1} \times[0,1]$ defined as follows: for each positive integer $n$ let

$$
\begin{aligned}
a_{n}^{k} & =\left(a_{n}^{k-1}, 1\right) \text { if } n \leqq k \\
& =\left(a_{n}^{k-1}, \frac{1}{2 n-1}\right) \text { if } n>k, \\
A_{n}^{k} & =A_{n}^{k-1} \times\{1\} \quad \text { if } n \leqq k,
\end{aligned}
$$

and if $n>k A_{n}^{k}$ is defined by recursion,

$$
\begin{aligned}
& A_{k+1}^{k}=A_{k}^{k} \cup\left(\left\{a_{k}^{k-1}\right\} \times\left[\frac{1}{2 k}, 1\right]\right) \cup\left(A_{k}^{k-1} \times\left\{\frac{1}{2 k}\right\}\right) \\
& \cup\left(\left\{1_{k-1}\right\} \times\left[\frac{1}{2 k+1}, \frac{1}{2 k}\right]\right) \cup\left(A_{k+1}^{k-1} \times\left\{\frac{1}{2 k+1}\right\}\right), \\
& \vdots \\
& A_{n}^{k}=A_{n-1}^{k} \cup\left(\left\{a_{n-1}^{k-1}\right\} \times\left[\frac{1}{2 n-2}, \frac{1}{2 n-3}\right]\right)
\end{aligned}
$$




$$
\begin{aligned}
& \cup\left(A_{n-1}^{k-1} \times\left\{\frac{1}{2 n-2}\right\}\right) \cup\left(\left\{1_{k-1}\right\} \times\left[\frac{1}{2 n-1}, \frac{1}{2 n-2}\right]\right) \\
& \cup\left(A_{n}^{k-1} \times\left\{\frac{1}{2 n-1}\right\}\right), \cdots .
\end{aligned}
$$

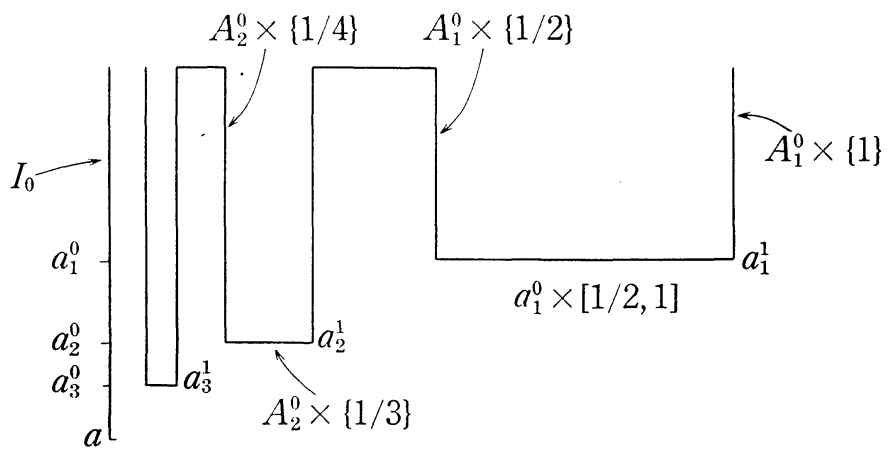

$I_{1}$

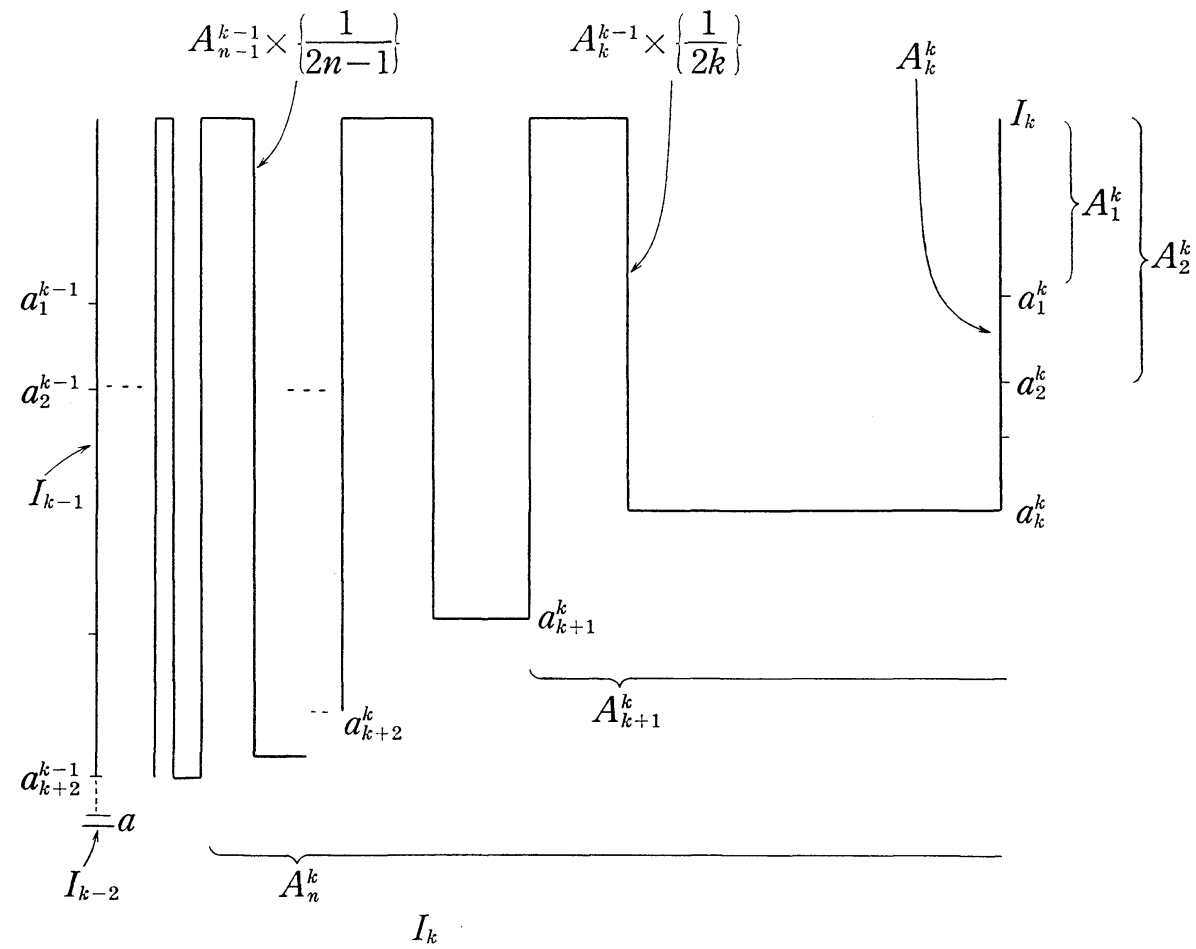

Then let $I_{k}=\left(I_{k-1} \times\{0\}\right) \cup \bigcup_{n=1}^{\infty} A_{n}^{k}, 1_{k}=\left(1_{k-1}, 1\right)$ and identify $I_{k-1}$ with $I_{k-1} \times\{0\}$ using the natural mapping; let $r_{k-1}^{k}$ be the projection of $I_{k}$ onto $I_{k-1}$. Thus $\left\{a_{n}^{k}\right\}_{n=1}^{\infty}$ converges to $a=(a, 0), A_{n}^{k} \subset A_{n+1}^{k}$, and $\operatorname{Cmps}\left(I_{k}, 1_{k}\right)=\bigcup_{n=1}^{\infty} A_{n}^{k}$. 
The following properties of the construction will be used in the proofs:

(P1) $I_{k}$ is irreducible from $1_{k}$ to $I_{k-1}$;

(P2) no point of $I_{k}-I_{k-1}$ is mapped by $r_{k-1}^{k}$ into $I_{k-2}$ and each point of $I_{k}-I_{k-1}$ is mapped into $\operatorname{Cmps}\left(I_{k-1}, 1_{k-1}\right)$;

(P3) for each $n$ and $\beta<\alpha r_{\beta}^{\alpha}\left(a_{n}^{\alpha}\right)=a_{n}^{\beta}$ and $r_{\beta}^{\alpha}\left(A_{n}^{\alpha}\right)=A_{n}^{\beta}$;

(P4) if $k \leqq n$ then $\pi_{n}\left(A_{k}^{n}\right)=\{1\}$, if $k>n$ then $\pi_{n}\left(A_{k}^{n}\right)=[1 /(2 k-1), 1]$, and $\pi_{n}^{-1}(1)=A_{n}^{n}$;

and

(P5) every point of $\left\{a_{k+1}^{k-1}\right\} \times[1 /(2 k-1), 1]$ separates $I_{k}$. Let $I_{\omega_{0}}=$ $\lim \left\{I_{n}, r\right\}_{n<\omega_{0}}$, and let $1_{\omega_{0}}$ be the point $x$ such that $x_{n}=1_{n}$. Then for each integer $n, I_{n}$ can be identified with $\lim \left\{I_{n}, r_{k}^{k+1}\right\}_{n<k<\omega_{0}}$ since $r_{k}^{k+1}$ is the identity on $I_{k}$ for $k>n$. Let $I_{n} \overleftarrow{\text { be }}$ so identified using the natural mapping. So $I_{n} \subset I_{0} \times \prod_{i=1}^{n}[0,1]$ and $I_{\omega_{0}}$ is identified with the subset $\overline{\bigcup_{n=1}^{\infty} I_{n}}$ of $I_{0} \times \prod_{i=1}^{\omega_{0}}[0,1]$. Further define $A_{i}^{\omega_{0}}$ for each positive integer $i$ by $A_{i}^{\omega_{0}}=\lim \left\{A_{i}^{n}, r\right\}_{n=1}^{\infty}$, property P3 insures that $A_{i}^{\omega_{0}}$ is well defined. Note that it follows from the construction that if $x \in I_{\omega_{0}}$ and $a<b$ then $\pi_{i}\left(x_{a}\right)=\pi_{i}\left(x_{b}\right)$ for all $i \leqq a$.

Claim 1. $I_{\omega_{0}}$ is indecomposable.

Proof. Suppose not and that $H$ and $K$ are two proper subcontinua of $I_{\omega_{0}}$ whose union is $I_{\omega_{0}}$. Then there exist open sets $R$ and $S$ such that $R \subset H \backslash K$ and $S \subset K \backslash H$ and hence are mutually exclusive. There exists an integer $j$ and two open sets $R_{j}$ and $S_{j}$ in $I_{j}$ such that $\overleftarrow{R_{j}} \subset R$ and $\overleftarrow{S}_{j} \subset S$. Since $I_{j}=\overline{\mathrm{U}_{n=1}^{\infty} A_{n}^{j}}$ there is an integer $i$ so that both $R_{j}$ and $S_{j}$ intersect $A_{i}^{j}$. Therefore $R_{j} \times[0,1]$ and $S_{j} \times$ $[0,1]$ both intersect $A_{i}^{j} \times\{1 /(2 i-1)\}$. So each of $\Pi_{j+1}(R)$ and $\Pi_{j+1}(S)$ intersect both $I_{j}$ and $A_{i}^{j+1}$, hence each of $\Pi(H)$ and $\Pi(K)$ intersect both $I_{j}$ and $A_{i}^{j+1}$. By the irreducibility of $I_{j+1}$ from $1_{j+1}$ to $I_{j}$ it follows that $I_{j}$ is a subset of both $\Pi_{j+1}(H)$ and $\Pi_{j+1}(K)$ (recall that $\left.I_{j}=I_{j} \times\{0\}\right)$ and hence $I_{j}=\Pi_{j}(H)=\Pi_{j}(K)$ which contradicts the fact that $\vec{R}_{j}$ and $\vec{S}_{j}$ must be mutually exclusive. Thus $I_{\omega_{0}}$ is indecomposable.

Claim 2. If $x \in I_{\omega_{0}}$ and there is a positive integer $j$ such that $\pi_{j}\left(x_{j}\right)=0$, then $\pi_{i}\left(x_{i}\right)=0$ for all $i>j$.

Proof. Suppose $x \in I_{\omega_{0}}, x_{\alpha} \in I_{\alpha}$ and $\pi_{\alpha}\left(x_{\alpha}\right) \neq 0$. Then there exists an integer $n$ such that $x_{\alpha} \in A_{n}^{\alpha}$. But $r_{\alpha-1}^{\alpha}\left(A_{n}^{\alpha}\right)=A_{n}^{\alpha-1}$ and either $\pi_{\alpha-1}\left(A_{n}^{\alpha-1}\right)=[1 /(2 n-1), 1]$ or $\pi_{\alpha-1}\left(A_{n}^{\alpha-1}\right)=1$, and in either case $\pi_{\alpha-1}\left(x_{\alpha-1}\right) \neq 0$ so $\pi_{\alpha-1}\left(x_{\alpha}\right) \neq 0$. So if $\pi_{j}\left(x_{j}\right)=0$ then $\pi_{j+1}\left(x_{j+1}\right)=0$ and the claim follows by induction. 
Claim 3. If $K$ is a proper subcontinuum of $I_{\omega_{0}}$ containing $1_{\omega_{0}}$ then there exists an integer $\beta$ so that if $\gamma>\beta$ then $\pi_{\alpha}\left(\Pi_{r}(K)\right)=1$ for all $\alpha$ so that $\beta<\alpha \leqq \gamma$.

Proof. Suppose that there is a proper subcontinuum $K$ of $I_{\omega_{0}}$ for which the claim is not true. Then if $\beta$ is an integer there exists an integer $\gamma>\beta$ so that $\pi_{r}\left(\Pi_{r}(K)\right)$ is nondegenerate. Suppose in addition that for each $\beta$ there is a $\gamma>\beta$ so that $\pi_{\gamma}\left(\Pi_{\gamma}(K)\right)=0$. Then by Claim 2 since $1 \in \pi_{\alpha}\left(\Pi_{\gamma}(K)\right)$ for all $\alpha<\gamma$ it follows that $I_{\gamma-1} \subset$ $\Pi_{r}(K)$. But then $K=I_{\omega_{0}}$ which is a contradiction. So the supposition is false and there exists an integer $b$ so that if $\gamma>b$ then $0 \notin$ $\pi_{\alpha}\left(\Pi_{\alpha}(K)\right)$ for all $\alpha$ such that $b<\alpha<\gamma$.

Suppose $B>b$, where $b$ is defined above. Then from the negation of the claim, for each positive integer $n$ there is an integer $\gamma_{n}$ with $\beta+n<\gamma_{n}$ so that $\pi_{\gamma_{n}}\left(\Pi_{\gamma_{n}}(K)\right)$ is nondegenerate. But then $\left(a_{r_{n}}^{\gamma_{n}-1}, 1\right) €$ $\Pi_{r_{n}}(K)$. So $\alpha_{\gamma_{n}}^{\beta} \in \Pi_{\beta}(K)$ (by P3), thus if $\gamma_{n}=\beta+k_{n}$ for some positive integer $k_{n}>n$ then $\alpha_{\beta+k_{n}}^{\beta} \in \prod_{r_{n}}(K)$ and thus $\alpha_{\beta+k_{n}}^{\beta} \in \Pi_{\beta}(K)$ (by P3). So there is unbounded sequence in $\left\{k_{n}\right\}_{n=1}^{\infty}$ so that $a_{\beta+k_{n}}^{\beta} \in \prod_{\beta}(K)$, but $a$ is the sequential limit of $\left\{a_{i}^{\beta}\right\}_{i=1}^{\infty}$ and hence is a limit point of the set $\left\{a_{\beta+k_{n}}^{\beta} \mid n\right.$ is a positive integer $\}$, so $a \in \Pi_{\beta}(K)$. Now $\pi_{\alpha}(a)=0$ for all $\alpha>1$ so $0 \in \pi_{\alpha}\left(\Pi_{\beta}(K)\right)$ for all $0<\alpha \leqq \beta$ which contradicts the choice of $\beta>b$. So the claim has been established.

Claim 4. $\operatorname{Cmps}\left(I_{\omega_{0}}, 1_{\omega_{0}}\right)=\bigcup_{i=1}^{\infty} A_{i}^{\omega_{0}}$.

Proof. Suppose $x \in \operatorname{Cmps}\left(I_{\omega_{0}}, 1_{\omega_{0}}\right)$. By Claim 3 there exists an integer $\beta$ so that if $\gamma>\beta$ and $\alpha$ is an integer so that $\beta<\alpha \leqq \gamma$ then $\pi_{\alpha}\left(x_{r}\right)=1$. Let $\gamma>\beta$, then $x_{r} \in A_{\gamma}^{\gamma}$ (by P4). Thus $x_{\alpha} \in r_{\alpha}^{\gamma}\left(A_{\gamma}^{\gamma}\right)$ and $\boldsymbol{r}_{\alpha}^{r}\left(\boldsymbol{A}_{r}^{r}\right)=\pi_{\alpha}\left(A_{r}^{\omega_{0}}\right)$. So $x \in A_{r}^{\omega_{0}}$. So Claim 4 has been established.

The construction of $I_{\mu}$ for $\mu$ an ordinal greater than $\omega_{0}$ follows. Suppose $\delta$ is a limit ordinal and that $\left\{A_{i}\right\}_{i=1}^{\infty},\left\{a_{i}\right\}_{i=1}^{\infty}, C_{\lambda}, r_{\beta}^{\lambda}$, and $I_{\lambda}$ have feen defined for all $\lambda \leqq \delta$ so that:

(1) For each positive integer $i$ the continuum $A_{i}^{\lambda}$ is irreducible from $a_{i}^{\lambda}$ to $1_{\lambda}$.

(2) $C_{\lambda}=\bigcup_{i=1}^{\infty} A_{i}^{\lambda}$.

(3) If $\beta<\lambda$ then $r_{\beta}^{\lambda}\left(A_{i}^{\lambda}\right)=A_{i}^{\beta}, r_{\beta}^{\lambda}\left(a_{i}^{\lambda}\right)=a_{i}^{\beta}$, and $\left\{a_{i}^{\lambda}\right\}_{i=1}^{\infty}$ converges to $a$.

(4) If $\beta<\lambda$ then $r_{\beta}^{\lambda}\left(I_{\lambda}-I_{\beta}\right)=C_{\beta}$.

(5) $C_{\lambda}=\operatorname{Cmps}\left(I_{\lambda}, 1_{\lambda}\right)=\left\{P \mid\right.$ there exists a $\beta<\delta$ such that $\pi_{\lambda}\left(P_{\lambda}\right)=$ 1 for all $\gamma>\beta$ \}.

Then construct $I_{\dot{0}+n}$ for all positive integers $n$ by substituting $I_{\delta}$ for $I_{0}, A_{i}^{\delta}$ for $A_{i}^{0}, a_{i}^{\delta}$ for $a_{i}^{0}$, and $1_{\delta}$ for $1_{0}$ in the construction of $I_{n}$ above. Compare condition 4 with a similar condition in Bellamy [1]. 
Suppose that $\mu$ is a limit ordinal and $I_{\gamma}$ has been defined for all $\gamma<\mu . \quad$ Let $I_{\mu}=\lim \left\{I_{\gamma}, r\right\}_{\gamma<\mu}, A_{i}^{\mu}=\lim \left\{A_{i}^{\gamma}, r\right\}_{\gamma<\mu}, a_{i}^{\mu}=\lim \left\{a_{i}, r\right\}_{\gamma<\mu}$, and for each $\beta<\mu \overleftarrow{\text { let }} r_{\beta}^{\prime \prime}$ be the projection of $I_{\mu}$ onto $I_{\beta}^{\leftarrow}$. As above identify $I_{r}$ with $\lim _{\leftarrow}\left\{I_{r}, r\right\}_{\gamma<\alpha<\mu}$ and $a$ with $\vec{a}$. The argument of Claim 1 can be used to prove that $I_{\mu}$ is indecomposable. Claim 2 also generalizes for $I_{\mu}$ as follows:

Claim 5. If $x \in I_{\mu}$ and there is an ordinal $j<\mu$ which is not a limit ordinal such that $\pi_{j}\left(x_{j}\right)=0$ then $\pi_{i}\left(x_{i}\right)=0$ for all ordinals $i, j<i<\mu$, which are not limit ordinals; and hence $x \in I_{j}$.

Proof. Suppose $x \in I_{\mu}$ and $j=\lambda+q$ for some limit ordinal $\lambda$ and positive integer $q$. If $\alpha=\lambda^{\prime}+r$ for some limit ordinal $\lambda^{\prime} \geqq \lambda$ with $\lambda^{\prime}+r>\lambda$ and $r>0$ and it is true that $\pi_{\alpha}\left(x_{\alpha}\right) \neq 0$, then there exists an integer $n$ so that $x_{\alpha} \in A_{n}^{\alpha}$. But $r_{j}^{\alpha}\left(A_{n}^{\alpha}\right)=A_{n}^{j}$ and either $\pi_{j}\left(A_{n}^{j}\right)=[1 /(2 n-1), 1]$ or $\pi_{j}\left(A_{n}^{j}\right)=1$ (by P4). In either case $\pi_{j}\left(x_{\alpha}\right) \neq$ 0 . But $\pi_{j}\left(x_{j}\right)=\pi_{j}\left(x_{\alpha}\right)$, so that $\pi_{j}\left(x_{j}\right) \neq 0$, which is a contradiction.

Claims 6,7 , and 8 are concerned with the continuum $I_{\mu}$.

Claim 6. If $K$ is a subcontinuum of $I_{\mu}$ and $a \in \Pi_{1}(K)$ then $a \in K$.

Proof. If $a \in \Pi_{1}(K)$ then $(a, 0) \in \Pi_{2}(K)$ so $a \in \Pi_{2}(K)$. From Claim 5 it follows that $a \in \prod_{r}(K)$ for all $\gamma \in \mu$ since $a$ is identified with $a \times\{0\}$. Thus $a$ must belong to $K$.

Claim 7.

$$
\operatorname{Cmps}\left(I_{\mu}, 1_{\mu}\right)=\bigcup_{i=1}^{\infty} A_{i}^{\mu}
$$

Proof. Suppose that $K$ is a proper subcontinuum of $I_{\mu}$ containing $1_{\mu}$. If it is true that there is an integer $n$ so that if $\gamma<\mu$ then $a_{n}^{\gamma} \notin \Pi_{\gamma}(K)$, then it would follow that $\Pi_{\gamma}(K) \subset A_{n}^{\prime \prime}$ for all $\gamma<\mu$, and so $K \subset A_{n}^{\mu}$. So suppose that this is not true. Thus for each integer $n$ there exists an ordinal $\gamma_{n}<\mu$ such that $a_{n}^{\gamma_{n}} \in \Pi_{\gamma_{n}}(K)$. But then $a_{n}^{1} \in \Pi_{1}(K)$ for all $n$, since $r_{1}^{r n}\left(a_{n}^{\gamma}\right)=a_{n}^{1}$. So $a \in \Pi_{1}(K)$ and $a \in K$ by Claim 6. But then $K=I_{n}$ since $I_{n}$ is irreducible from $a$ to $1_{\mu}$. So the claim is true.

Claim 8. $I_{\mu}$ satisfies the following for each ordinal $\beta, \beta<\mu$, and each positive integer $i$ :

(1) $A_{i}^{\mu}$ is irreducible from $a_{i}^{\mu}$ to $1_{\mu}$.

(2) $C=\operatorname{Cmps}\left(I_{\mu}, 1_{\mu}\right)=\bigcup_{i=1}^{\infty} A_{i}^{\mu}$.

(3) $r_{\beta}^{\mu}\left(A_{i}^{\mu}\right)=A_{i}^{\beta}, r_{\beta}^{\prime \prime}\left(a_{i}^{\mu}\right)=a_{i}^{\beta}$, and $\left\{a_{i}^{\mu}\right\}_{i=1}^{\infty}$ converges to $a$. 


\section{(4) $r_{\beta}^{\prime \prime}\left(I_{n}-I_{\beta}\right)=C_{\beta}$.}

Proof. Part (1) follows from the irreducibility of $\Pi_{r}\left(A_{i}^{\mu}\right)$ for each $\gamma<\mu$, and part (2) follows from Claim 7. Since for each ordinal $\gamma<\mu$ the sequence $\left\{\prod_{r}\left(a_{i}^{\mu}\right)\right\}_{i=1}^{\infty}$ converges to $a$, it follows that $\left\{a_{i}^{\mu}\right\}_{i=1}^{\infty}$ converges to $\bar{a}$ which is identified with $a$. The rest of (3) follows from the definitions of $r_{\beta}^{\mu}, A_{i}^{\beta}$, and $a_{i}^{\beta}$. To prove (4) suppose that $x \in I_{t^{l}}-I_{\beta}$. Then by Claim $5, \pi_{\beta+1}\left(x_{\beta+1}\right) \neq 0$ so $x_{\beta+1} \in A_{n}^{\beta+1}$ for some integer $n$, but $r_{\beta}^{\beta+1}\left(A_{n}^{\beta+1}\right) \subset C_{\beta}$, thus $r_{\beta}^{\beta+1}\left(x_{\beta+1}\right) \in C_{\beta}$ so $r_{\beta}^{\mu}(x) \in C_{\beta}$; equality follows from parts (2) and (3).

Claim 9. The continuum $I_{\omega_{1}}=\lim _{\longleftarrow}\left\{I_{\lambda}, r\right\}_{\lambda<\omega_{1}}$ has exactly two composants.

Proof. From the construction, $\left\{I_{\gamma}\right\}_{r<\omega_{1}}$ is a monototic collection of continua. (a) If $\beta>\gamma$ then $I_{\gamma}$ does not intersect $C_{\beta}$ because $I_{\gamma}$ does not intersect $C_{\gamma+1}$ and if $\beta>\gamma, C_{\gamma+1}=r_{\gamma+1}^{\beta}\left(C_{\beta}\right)$. (b) From (4) of Claim 8 it follows that $r_{\beta}^{\alpha}\left(I_{\alpha}-I_{\beta}\right)=C_{\beta}$ for $\alpha>\beta$. Let $W=\{x \mid$ there is a $\gamma$ so that if $\alpha>\gamma$ then $\left.\pi_{\alpha}\left(x_{\alpha}\right)=0\right\}$. If $x \in W$ and $\gamma$ is the ordinal specified in the definition of $W$ then $x \in I_{r}$. So $x$ lies in the same composant as $a$.

Now $I_{\omega_{1}}$ is irreducible from $a$ to $1_{\omega_{1}}$, it will now be shown that if $y$ is a point of $I_{\omega_{1}}$ not in $W$ then $y$ lies in $\operatorname{Cmps}\left(I_{\omega_{1}}, 1_{\omega_{1}}\right)$. Suppose $y \notin W$. The following two conditions need to be established: (i) if $\alpha>\beta$ then $y_{\alpha} \notin I_{\beta}$, and (ii) $y_{\alpha} \in C_{\alpha}$. If $\alpha>\beta$ there exists an ordinal $\delta>\alpha$ such that $y_{\alpha} \neq y_{o}$ or else $y \in W$ (in particular $y \in I_{\alpha}$ ). Suppose that $y_{\alpha} \in I_{\beta}$, then $y_{\alpha} \notin C_{\alpha}$ by (a) above. But $r_{\alpha}^{\delta}\left(I_{\delta}-I_{\alpha}\right) \subset C_{\alpha}$ so $y_{i} \notin$ $I_{\hat{o}}-I_{\alpha}$, so $y_{\hat{o}} \in I_{\alpha}$. But $\left.r_{\alpha}^{\dagger}\right|_{I_{\alpha}}$ is the identity which contradicts the fact that $y_{j} \neq y_{\alpha}$. Thus (i) has been shown, also it has been shown that if $\alpha>\beta$ then there exists a $\delta>\alpha$ such that $y_{\delta} \notin I_{\alpha}$. So $y_{\delta} \in$ $I_{\hat{o}}-I_{\alpha}, r_{\alpha}^{i}\left(I_{\delta}-I_{\alpha}\right) \subset C_{\alpha}$, and so (ii) has been shown.

Suppose that $y \notin W$. By (i) if $\alpha>1$ then $y_{\alpha} \notin I_{1}$, and by (ii) $y_{\alpha} \in C_{\alpha}$. Thus by (2) of Claim 8 there exists an integer $n_{\alpha}$ so that $y_{\alpha} \in A_{n_{\alpha}}^{\alpha}$. There exists an uncountable subset $J$ of $\omega_{1}$ and an integer $n$ so that $n_{\alpha}=n$ for all $\alpha \in J$. But since $r_{\beta}^{\alpha}\left(A_{n}^{\alpha}\right)=A_{n}^{\beta}$ it follows that $y \in \lim \left\{A_{n}^{\beta}, r\right\}_{\beta<\omega_{1}}$ which is a proper subcontinuum of $I$ containing $1_{\omega_{1}}$. Thus it has been shown that if $y \notin W$ then $y \in \operatorname{Cmps}\left(I_{\omega_{1}}, 1_{\omega_{1}}\right)=C_{\omega_{1}}$. So $I_{\omega_{1}}$ has exactly two composants $W$ and $C_{\omega_{1}}$.

One can see that $X$ is a retract of each $I_{n}$ and hence of $I_{\omega_{1}}$. In order to construct a continuum with only one composant which has $X$ as a retract it is only necessary to construct $I_{0}$ and a retraction $r$ from $I_{0}$ onto $X$ that maps $1_{0}$ onto $a$, then by identifying $a$ and the point $1_{\omega_{1}}$ the continuum $I_{\omega_{1}}$ satisfies the desired condition. 


\section{REFERENCES}

1. D. P. Bellamy, Indecomposable continua with one and two composants, to appear.

2. G. R. Gordh,, Jr., Every continuum is a retract of some irreducible indecomposable continuum, Colloquia Mathematica Societaties Janos Bolyai, 8 (1972), 347-350.

3. R. L. Moore, Foundations of Point Set Theory, Amer. Math. Soc. Colloq. Pub. XIII, Revised edition, Providence, R. I.

4. Michel Smith, Generating large indecomposable continua, Pacific J. Math., 62 (1976), 587-593.

Received December 8, 1976 and in revised form June 12, 1979.

AUBuRN UNIVERSITY

AUBURN, AL 36830 


\title{
PACIFIC JOURNAL OF MATHEMATICS
}

\section{EDITORS}

\author{
DoNALD BABBITT (Managing Editor) \\ University of California \\ Los Angeles, CA 90024 \\ HUGo RossI \\ University of Utah \\ Salt Lake City, UT 84112 \\ C. C. MOORE and ANDREW OGG \\ University of California \\ Berkeley, CA 94720
}

J. DugundJI

Department of Mathematics

University of Southern California

Los Angeles, CA 90007

R. FINN and J. Milgram

Stanford University

Stanford, CA 94305

\section{ASSOCIATE EDITORS}
E. F. BeCKenbach
B. H. NeumanN
F. WOLF
K. YoshidA

\section{SUPPORTING INSTITUTIONS}

\author{
UNIVERSITY OF BRITISH COLUMBIA \\ CALIFORNIA INSTITUTE OF TECHNOLOGY \\ UNIVERSITY OF CALIFORNIA \\ MONTANA STATE UNIVERSITY \\ UNIVERSITY OF NEVADA, RENO \\ NEW MEXICO STATE UNIVERSITY \\ OREGON STATE UNIVERSITY \\ UNIVERSITY OF OREGON
}

\author{
UNIVERSITY OF SOUTHERN CALIFORNIA \\ STANFORD UNIVERSITY \\ UNIVERSITY OF HAWAII \\ UNIVERSITY OF TOKYO \\ UNIVERSITY OF UTAH \\ WASHINGTON STATE UNIVERSITY \\ UNIVERSITY OF WASHINGTON
}

The Supporting Institutions listed above contribute to the cost of publication of this Journal, but they are not owners or publishers and have no responsibility for its content or policies.

Mathematical papers intended for publication in the Pacific Journal of Mathematics should be in typed form or offset-reproduced, (not dittoed), double spaced with large margins. Please do not use built up fractions in the text of the manuscript. However, you may use them in the displayed equations. Underline Greek letters in red, German in green, and script in blue. The first paragraph or two must be capable of being used separately as a synopsis of the entire paper. Please propose a heading for the odd numbered pages of less than 35 characters. Manuscripts, in triplicate, may be sent to any one of the editors. Please classify according to the scheme of Math. Reviews, Index to Vol. 39. Supply name and address of author to whom proofs should be sent. All other communications should be addressed to the managing editor, or Elaine Barth, University of California, Los Angeles, California, 90024.

50 reprints to each author are provided free for each article, only if page charges have been substantially paid. Additional copies may be obtained at cost in multiples of 50 .

The Pacific Journal of Mathematics is issued monthly as of January 1966. Regular subscription rate: $\$ 84.00$ a year $(6$ Vols., 12 issues). Special rato: $\$ 42.00$ a year to individual members of supporting institutions.

Subscriptions, orders for numbers issued in the last three calendar years, and changes of address shoud be sent to Pacific Journal of Mathematics, P.O. Box 969, Carmel Valley, CA 93924, U.S.A Old back numbers obtainable from Kraus Periodicals Co., Route 100, Millwood, NY 10546.

\section{PUBLISHED BY PACIFIC JOURNAL OF MATHEMATICS, A NON-PROFIT CORPORATION}

Printed at Kokusai Bunken Insatsusha (International Academic Printing Co., Ltd.). 8-8, 3-chome, Takadanobaba, Shinjuku-ku, Tokyo 160, Japan. 


\section{Pacific Journal of Mathematics}

\section{Vol. 86, No. 2 December, 1980}

Graham Donald Allen, David Alan Legg and Joseph Dinneen Ward, Hermitian

liftings in Orlicz sequence spaces ............................... 379

George Bachman and Alan Sultan, On regular extensions of measures ........ 389

Bruce Alan Barnes, Representations Naimark-related to $*$-representations; a

correction: "When is a representation of a Banach $*$-algebra

Naimark-related to a $*$-representation?" ........................ 397

Earl Robert Berkson, One-parameter semigroups of isometries into $H^{p} \ldots \ldots .403$

M. Brodmann, Piecewise catenarian and going between rings ............ 415

Joe Peter Buhler, A note on tamely ramified polynomials ............... 421

William Lee Bynum, Normal structure coefficients for Banach spaces ........ 427

Lung O. Chung, Biharmonic and polyharmonic principal functions ......... 437

Vladimir Drobot and S. McDonald, Approximation properties of polynomials

with bounded integer coefficients .............................

Giora Dula and Elyahu Katz, Recursion formulas for the homology of

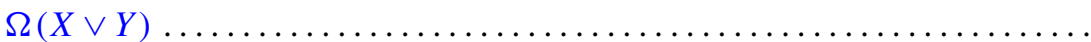

John A. Ernest, The computation of the generalized spectrum of certain Toeplitz operators ...................................... 463

Kenneth R. Goodearl and Thomas Benny Rushing, Direct limit groups and the

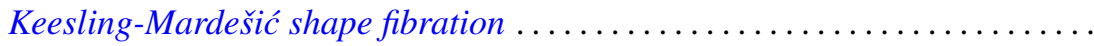

Raymond Heitmann and Stephen Joseph McAdam, Good chains with bad contractions

Patricia Jones and Steve Chong Hong Ligh, Finite hereditary near-ring-semigroups .

Yoshikazu Katayama, Isomorphisms of the Fourier algebras in crossed

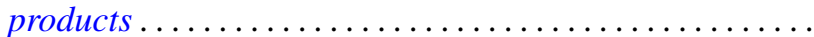

Meir Katchalski and Andrew Chiang-Fung Liu, Symmetric twins and common transversals. .

Mohammad Ahmad Khan, Chain conditions on subgroups of LCA groups ....

Helmut Kröger, Padé approximants on Banach space operator equations ...

Gabriel Michael Miller Obi, An algebraic extension of the Lax-Milgram theorem . .

S. G. Pandit, Differential systems with impulsive perturbation .

Richard Pell, Support point functions and the Loewner variation ...

J. Hyam Rubinstein, Dehn's lemma and handle decompositions of some

4-manifolds

James Eugene Shirey, On the theorem of Helley concerning finite-dimensional subspaces of a dual space.

Oved Shisha, Tchebycheff systems and best partial bases.... 